\title{
Prediction of quality of life after stroke rehabilitation
}

\author{
Jiann-Der Lee ${ }^{1,2,3}$, Tzyh-Chyang Chang ${ }^{1,4}$, Shih-Ting Yang ${ }^{1}$, Chung-Hsien Huang ${ }^{1}$, Fu-Han Hsieh ${ }^{+, 5,67}$, Ching-Yi \\ $\mathrm{Wu}^{+, 5,67}$
}

\section{ABSTRACT}

Introduction: The purpose of this study was to develop a computational method to identify potential predictors for quality of life (QOL) after post stroke rehabilitation.

Methods: Five classifiers were trained by five personal factors and nine functional outcome measures by 10 -fold cross-validation. The classifier with the highest cross-validated accuracy was considered to be the optimal classifier for QOL prediction.

Results: Particle Swarm-Optimized Support Vector Machine (PSO-SVM) showed highest accuracy in predicting QOL in stroke patients and was adopted as the optimal classifier. Potential predictors were assessed by PSO-SVM with feature selection. The early outcomes of Quality of Movement scale of the Motor Activity Log (MAL_QOM) and the Stroke Impact Scale (SIS) were identified to be the most predictive outcome predictors for QOL.

Conclusion: The approach provides the medical team another possibility to improve the accuracy in predicting QOL in stroke patients. Therapists could determine the therapies for stroke patients more accurately and efficiently to enhance the quality of life after stroke.

\section{Keywords}

Stroke, Quality of life, Outcome prediction, Classifier

\section{Introduction \\ Stroke remains a leading cause of death and disability in the developed world [1]. After stroke, the effects of stroke and post stroke rehabilitation are usually assessed by health professional ratings and performance tests [2-4]. However, real life of stroke survivors is affected in multiple ways and may not be described completely by only}

health and functional status. It is possible that a treatment succeeds in enhancing physical function recovery however induces psychosocial problems [5,6]. In this case, quality of life (QOL) may actually be degraded after poststroke rehabilitation. The WHO suggests that a comprehensive view of quality of life includes not only physical health, but also psychological

\footnotetext{
'Department of Electrical Engineering, Chang Gung University, Taoyuan 333, Taiwan

2Department of Neurosurgery, Chang Gung Memorial Hospital at LinKou, Taoyuan 333, Taiwan

3Department of Electrical Engineering, Ming Chi University of Technology, New Taipei City 24301, Taiwan

${ }^{4}$ Department of Occupational Therapy, Bali Psychiatric Center, New Taipei City 249, Taiwan

${ }^{5}$ Department of Occupational Therapy, Chang Gung University, Taoyuan 333, Taiwan

${ }^{6}$ Healthy Aging Research Center, Chang Gung University, Taoyuan 333, Taiwan

'Department of Physical Medicine and Rehabilitation, Chang Gung Memorial Hospital at Linkou, Taoyuan, Taiwan

${ }^{\dagger}$ Author for correspondence: Fu-Han Hsieh, Department of Occupational Therapy and Graduate Institute of Behavioral Sciences, College of Medicine, Chang Gung University, Tao-Yuan, 333, Taiwan;

Telephone: +886-3-2118800, ext: 3355, 5761, email: fuhan@mail.cgu.edu.tw

${ }^{+}$Author for correspondence: Ching-Yi Wu, Department of Occupational Therapy and Graduate Institute of Behavioral Sciences, College of Medicine, Chang Gung University, Tao-Yuan, 333, Taiwan; Healthy Aging Research Center, Chang Gung University, Tao-Yuan, 333, Taiwan; Telephone: +886-3-2118800, ext: 3355, 5761, email: cywu@mail.cgu.edu.tw
} 
health, social relationships, and environmental quality [4]. Therefore, to obtain a comprehensive view of the effects after stroke, life quality should also be considered when assessing a person's health and functioning.

In recent years, assessment of QOL in stroke has become increasingly common. Many recent rehabilitation therapies have been reported to be effective in restoring upper limb motor function after stroke but showed varied effects in QOL [7-10]. Different rehabilitation therapies may benefit different subgroups of the stroke population and cause different effects to QOL. Identifying key predictors of QOL may assist therapists to determine an optimal therapy, which can not only improve physical function but also maximize QOL for a specific subgroup of stroke survivors. Decision making of rehabilitation strategies may be more efficient and complete with identifying predominant predictors of QOL.

Only three studies examined predictors of QOL $[5,11,12]$. In these three studies, the predictive ability of multiple factors was examined, including demographic factors, vascular risk factors, clinical scales and neuropsychological assessment, and lesion characteristics. However, general predictors of outcomes of QOL were hard to determine because of the heterogeneity among these studies. Both physical and psychological factors were reported to be important in predicting QOL after stroke $[5,11,12]$. Although stroke rehabilitation gains in QOL are important, the question of which patients may benefit most in QOL from specific therapies has not been widely addressed, and statistical approaches to reveal such associations and predictors may not be optimal $[13,14]$. However, possible predictors related to QOL performance outcome after rehabilitation remained less discussed. More studies are needed to clarify the predictive ability of diverse QOL predictors in stroke patients.

Practical implementation of outcome predictors in clinical use was also constrained by the complexity of the algorithms. Developing prognostic algorithm based on existing and simple algorithms may reduce the complexity in clinical implementation, increase the use of prognostic model, and further improve the efficiency of rehabilitation therapy. Traditionally, studies examined outcome predictors used regression analysis to discriminate the most predictive factors from others [15-18]. However, the results of regression analysis can only explained the variance of the outcome in percentage. Computational methods can provide another aspect of outcome prediction. The results of regression statistical method showed that the factors were predictors for the outcome measure model, and the model only explained how percentage of the variance in the outcome measure scores. However, the results of computational classifier methods can provide accuracy and more application related to the predictors.

It has been applied in predicting clinical outcome in cancer patients and showed high accuracy and efficiency [19,20]. Using classifiers could improve the accuracy in predicting QOL. Hopefully, predominant predictors could also be better identified. That's why we try to utilize a computational classifier method to identify potential predictors for quality of life (QOL) after post stroke rehabilitation.

\section{Methods}

This study was a secondary analysis of data from previous $[7,8,10]$ and ongoing randomized controlled trials of stroke rehabilitation therapies. Institutional review board approval was obtained from Chang Gung Memorial Hospital. Written informed consent was obtained from each patient before study participation.

\section{- Participants}

A total of 130 stroke patients $(93$ men, 37 women) with a mean age of 54.96 years old (from 26 to 79 years old) were recruited from 2 participating sites. The patients were a mean of 20.98 months (from 2 to 68 months) after onset. All patients received independent examinations by physical therapists and occupational therapists to determine their eligibility for inclusion. The criteria for participants were: (1) Brunnstrom stage 3 or above for proximal and distal part of the affected upper limb [21], (2) no serious cognitive deficits (score $\geq 23$ on the MiniMental State Examination) [22], (3) no excessive spasticity (Modified Ashworth Scale $\leq 2$ at any joint of the affected upper limb) [23].

\section{- Design and intervention}

All subjects received individualized, treatment programs for 1.5-2 hours every day, 5 days per week for 3-4 weeks. Functional tasks used in therapy were selected by patients and therapists' joint decision. The functional tasks were including opening a drawer, picking up a cup and drinking 
from it, picking up a phone, and other functional movements simulating activities in daily living. Before and after the 3-4 week intervention period, the tests were administered by blinded raters. All participants in this study completed the pretest and posttest of all measures. Before administration of clinical measures, the blinded raters were trained to properly administer these measures. This training included careful review of written instructions and repeated practice. Rater competence was assessed by a senior certified occupational therapist.

\section{- Outcome measurement}

The Stroke Impact Scale (SIS) is a comprehensive measure of health-related quality of life in stroke patients [24-26] and is adopted as the certified outcome measure to determine the QOL of stroke patients in this study.

The SIS was adopted as the certified outcome measure for assessing QOL after stroke in this study. It has been evaluated as a reliable, valid, and sensitive outcome measure for measuring meaningful functional outcomes and healthrelated quality of life in stroke patients [23-26]. Version 3.0 of SIS comprises 59 items that assess eight domains: strength, hand function, activities and instrumental activities of daily living (ADL/ IADL), mobility, communication, emotion, memory and thinking, and participation. Patients responded to items in each domain using a 5-point rating scales.

The concept of minimal clinically important difference (MCID) was used to determine the smallest change in QOL. The MCID has been advocated to signify an important difference in patient symptoms and responsiveness.

Responsiveness refers to the ability of an instrument to detect the efficacy of a treatment. It can be conceptualized as longitudinal validity, indicating longitudinal change over a defined interval during or after treatment [27]. Jaeschke [28] first defined an MCID as being "the smallest difference in score in the domain of interest which patients perceive as beneficial.” Since then, the definition has varied. The MCID helps to define a threshold that is considered to be an important improvement.

In our previous study, MCID values for the full SIS version 3.0 are 9.2 for strength, 5.9 for ADL/IADL, 4.5 for mobility, and 17.8 for hand function [29]. The MCIDs of the other four domains of SIS (communication, emotion, memory and thinking, and participation) have not been reported in the literature. A change greater than $10 \%$ in SIS score were considered to be clinically meaningful improvement of these four domains of SIS based on clinical experience and consultation with physical therapists and stroke neurologists [9,30].

\section{- Predictors}

Fourteen parameters, including five personal factors and nine early functional outcomes, were adopted as potential outcome predictors for QOL. Personal factors include: age, gender, time since onset of injury, education attainment, and score of Mini-Mental State Evaluation (MMSE). Early functional outcomes include: Wolf Motor Function Test (WMFT), Fugl-Meyer Assessment (FMA), Functional Independence Measure (FIM), Motor Activity Log (MAL, including the amount of use scale: MAL_AOU, and the quality of movement scale: MAL_QOM), Action Research Arm Test (ARAT), ABILHAND, Physical Function, and Stoke Impact Scale (SIS).

\section{- Classification}

Five common classifiers were adopted in this study, including Back Propagation Artificial Neural Network (BP-ANN), Learning Vector Quantization (LVQ), Self-Organizing Mapping (SOM), Support Vector Machine (SVM), and Particle Swarm-Optimized SVM (PSO-SVM). First, the whole data set of these fourteen predictors was used as the input data for classifiers. The QOL after rehabilitation could be measured by SIS in this study. That's why the SIS was selected to be the outcome measure of the QOL after rehabilitation.

Comparing classified SIS scores with recorded SIS scores, predicting accuracy of the classifiers can be obtained. 10-fold cross-validation was applied to confirm the accuracy of each classifier. The classifier with the highest crossvalidated accuracy was considered to be the optimal classifier for QOL prediction. Two most commonly used feature selection algorithms [31], sequential forward selection (SFS) and sequential backward selection (SBS), were applied with the classifier to improve the accuracy and reduce required computing power for constructing the classifier. The processes were showed as a flowchart in Figure 1.

\section{Results}

\section{- Determining the optimal classifier}

Table 1 showed the performance of the five 


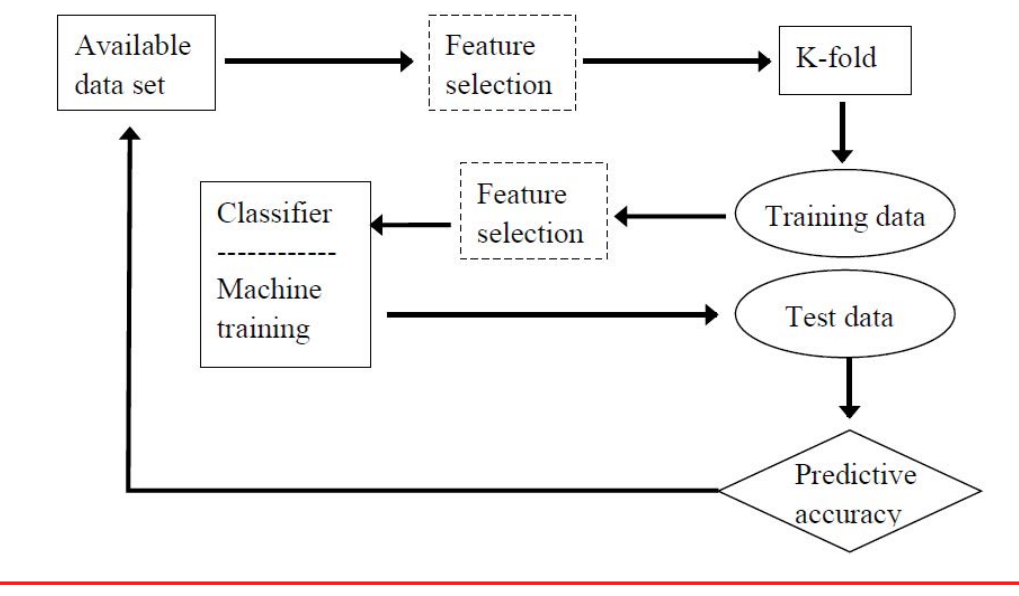

Figure 1: The procedure of classification of functional outcome predictors of QOL.

classifiers (BP-ANN, LVQ, SOM, SVM, and PSO-SVM). PSO-SVM showed the highest accuracy $(58.33 \%)$ and the highest crossvalidated accuracy (74.29\%) in predicting SIS outcome among all classifiers. PSO-SVM is likely to be the optimal classifier for identifying predictors of QOL in stroke patients.

\section{- Predictor classification}

Accuracy and cross-validated accuracy in predicting QOL of all chosen factors after PSOSVM with SFS were showed in Table 2.

In the following PSO-SVM model test, by using SFS method, we tried to add in one feature each time to see if the accuracy and cross validation would change or not. Table 2 showed the comparison of the classification results of adding in one feature each time which were chosen in PSO-SVM model.

In Table 2, the significant better cross validation results in SFS method are measure (D1) Age, (4) FIM, (6) MAL_QOM, (8) Physical Function and (9) SIS. At the same, the accuracy results of (D1) Age, (6) MAL_QOM, (8) Physical Function and (9) SIS are good might be considered as potential predictors. In addition, the accuracy results of (4) FIM is poor might not be considered as potential predictors. The (D1) Age, (6) MAL_QOM, (8) Physical Function and (9) SIS seemed to be the potential predictors of QOL performance because of higher accuracy rate. We chose these potential predictors: including (D1) Age, (6) MAL_QOM, (8) Physical Function and (9) SIS in following process to identify the appropriate predictors for QOL performance outcome after rehabilitation by using SFS method.

The SIS, age, Physical function, MAL_QOM, and FIM showed highest cross-validated accuracy (accuracy > 75\%). Among them, SIS, age, Physical function, and MAL_QOM had higher accuracy (accuracy $=60 \%$ ) than FIM (accuracy $=58.33 \%)$. Therefore, FIM was dismissed from the list of predominant predictors. As a result, SIS, age, Physical function, and MAL_QOM were considered to be predominant predictors for predicting final QOL.

To determine the priority of the predictors, combinations of these four predominant predictors were again assessed by PSO-SVM. The accuracy of each combination was presented in Table 3. The combination of MAL_QOM and SIS showed highest accuracy (70\%) and highest cross-validated accuracy (81.43\%). The early outcomes of MAL_QOM and SIS are likely to be predominant predictors for QOL.

These fourteen predictors were again assessed by PSO-SVM with SBS algorithm. When all predictors were included as the training set, the accuracy was $58.33 \%$ and the cross-validated accuracy was $74.29 \%$. When all five personal factors (D1 - D5 in Table 2) were excluded from the training set, the accuracy of the classification was still $58.33 \%$, and the cross-validated accuracy was still $74.29 \%$. Excluding personal factors did not affect the accuracy of QOL prediction. Hence, personal factors were considered not to be significant predictors for QOL. When WMFT was excluded from the predictor list, the accuracy of classification dropped to $55.00 \%$. For this reason, although the accuracy of QOL prediction based on early outcome of WMFT was relatively low when assessed by PSO-SVM with SFS, WMFT outcome may still have significant contribution to QOL prediction. When other predictors were excluded from the list individually, the accuracy was not much altered (close to $58.33 \%$ ). After evaluated by PSO-SVM with SBS, WMFT outcome was also considered as a predominant predictor for QOL.

Three identified predominant outcome predictors by PSO-SVM with SFS and SBS (SIS, MAL QOM, and WMFT) were again assessed by PSOSVM in different combinations to determine the optimal predictive model. The results were listed in Table 4. The combination of early outcomes of MAL_QOM and SIS showed highest accuracy $(70 \%)$ and highest cross-validated accuracy $(81.43 \%)$ in predicting final QOL.

\section{Discussion}

This study developed a computational method to 


\begin{tabular}{|c|c|c|}
\hline Classifier & Accuracy & $\begin{array}{l}\text { Cross-validated } \\
\text { accuracy }\end{array}$ \\
\hline $\begin{array}{l}\text { Back Propagation } \\
\text { Artificial Neural } \\
\text { Network (BP-ANN) }\end{array}$ & $38.33 \%$ & $48.51 \%$ \\
\hline $\begin{array}{l}\text { Learning Vector } \\
\text { Quantization (LVQ) }\end{array}$ & $50.00 \%$ & $58.96 \%$ \\
\hline $\begin{array}{l}\text { Self-Organizing } \\
\text { Mapping (SOM) }\end{array}$ & $53.33 \%$ & $66.57 \%$ \\
\hline $\begin{array}{l}\text { Support Vector } \\
\text { Machine (SVM) }\end{array}$ & $53.33 \%$ & $71.47 \%$ \\
\hline $\begin{array}{l}\text { Particle Swarm } \\
\text { Optimization SVM } \\
\text { (PSO-SVM) }\end{array}$ & $58.33 \%$ & $74.29 \%$ \\
\hline
\end{tabular}

Table 2: The performance of PSO-SVM with
SFS trained on different predictors.
\begin{tabular}{|c|c|c|}
\hline Measures & Accuracy & Average accuracy \\
\hline (D1) Age & $60 \%$ & $77.14 \%$ \\
\hline (D2) Gender & $58.33 \%$ & $74.29 \%$ \\
\hline (D3) Onset & $58.33 \%$ & $74.29 \%$ \\
\hline (D4) MMSE & $58.33 \%$ & $74.29 \%$ \\
\hline $\begin{array}{c}\text { (D5) Education } \\
\text { attainment }\end{array}$ & $58.33 \%$ & $74.29 \%$ \\
\hline (1) FMA & $8.33 \%$ & $74.29 \%$ \\
\hline (2) WMFT & $58.33 \%$ & $74.29 \%$ \\
\hline (3) ARAT & $58.33 \%$ & $74.29 \%$ \\
\hline (4) FIM & $58.33 \%$ & $75.71 \%$ \\
\hline (5) MAL_AOU & $58.33 \%$ & $74.29 \%$ \\
\hline (6) MAL_QOM & $60 \%$ & $75.71 \%$ \\
\hline (7) ABILHAND & $58.33 \%$ & $74.29 \%$ \\
\hline (8) Physical Function & $60 \%$ & $77.14 \%$ \\
\hline (9) SIS & $60 \%$ & $81.43 \%$ \\
\hline
\end{tabular}

Table 3: The performance of PSO-SVM with

SFS trained on combinations of predominant predictors.

\begin{tabular}{|c|c|c|}
\hline $\begin{array}{c}\text { Combination of } \\
\text { predictors }\end{array}$ & Accuracy & Average accuracy \\
\hline$(\mathrm{D} 1)+(6)+(8)+(9)$ & $58.33 \%$ & $74.29 \%$ \\
\hline$(\mathrm{D} 1)+(6)+(8)$ & $58.33 \%$ & $74.29 \%$ \\
\hline$(\mathrm{D} 1)+(6)+(9)$ & $63.33 \%$ & $80.00 \%$ \\
\hline$(\mathrm{D} 1)+(8)+(9)$ & $58.33 \%$ & $74.29 \%$ \\
\hline$(\mathrm{D} 1)+(6)$ & $60 \%$ & $77.14 \%$ \\
\hline$(\mathrm{D} 1)+(8)$ & $58.33 \%$ & $74.29 \%$ \\
\hline$(\mathrm{D} 1)+(9)$ & $60 \%$ & $77.14 \%$ \\
\hline$(6)+(8)+(9)$ & $58.33 \%$ & $74.29 \%$ \\
\hline$(8)+(9)$ & $60 \%$ & $77.14 \%$ \\
\hline$(6)+(8)$ & $58.33 \%$ & $74.29 \%$ \\
\hline$(6)+(9)$ & $70 \%$ & $81.43 \%$ \\
\hline
\end{tabular}

predict post-treatment QOL in stroke patients. Potential predictors were classified by five common classifiers into different levels of QOL (different SIS score). 10-fold cross-validation was applied to confirm the accuracy. The classifier
Table 4: The performance of PSO-SVM trained on WMFT, MAL_QOM, and SIS [(2), (6), and (9) in Table 2, respectively].

\begin{tabular}{|c|c|c|}
\hline $\begin{array}{c}\text { Combination of } \\
\text { predictors }\end{array}$ & Accuracy & Average accuracy \\
\hline$(2)+(6)+(9)$ & $56.67 \%$ & $75.71 \%$ \\
\hline$(2)+(6)$ & $58.33 \%$ & $74.29 \%$ \\
\hline$(2)+(9)$ & $53.33 \%$ & $74.29 \%$ \\
\hline$(6)+(9)$ & $70 \%$ & $81.43 \%$ \\
\hline
\end{tabular}

with the highest accuracy and cross-validated accuracy was considered to be the optimal classifier for QOL prediction. PSO-SVM showed the best performance in QOL predicting and was adopted as the classifier for QOL prediction in this study. Early functional outcome of MAL QOM and SIS were identified as predominant predictors for QOL in stroke patients.

To sum up, all comparison results for cross validation and accuracy of the five classifiers, PSO-SVM classifier get better performance (cross validation $=74.29 \%$, accuracy rate $=58.33 \%$ ) when all predictors are induced in making these categories among all these five classifiers. That's why PSO-SVM was chosen in this study to find the predictor of QOL performance for clients with stroke.

PSO-SVM classifier gets the results of the highest accuracy rate when all predictors are induced in making these categories among all five classifiers. And PSO-SVM was chosen in this study to find the predictor of QOL performance for clients with stroke.

PSO-SVM showed the best accuracy in predicting QOL. In previous study examined the accuracy of classifiers in feature selection, PSOSVM was demonstrated to be able to increase classification accuracy and minimize required computational resources [32]. Although PSOSVM was successful in predicting QOL in this study, classifying predictors with larger number of patients and more types of treatment is needed to evaluate the approach more thoroughly. Previous studies examined feature selection method suggested adaptive floating search method could improve the performance of classifiers [33,34]. For future works, other feature selection algorithms, such as adaptive floating search method, could be tested with PSO-SVM to improve the accuracy/efficiency of outcome prediction.

All personal factors, including age, gender, time since onset of injury, education attainment, and score of Mini-Mental State Evaluation were not 
significant predictors of QOL. Other studies have found similar findings $[5,35]$.

The combination of early outcomes of MAL_ QOM and SIS was identified as the optimal predictor for QOL. Both MAL_QOM and SIS involve measurement of participation in real world activities [36], indicating activities of daily living (ADL) could be an important index in predicting QOL outcome. In a previous study examined disablement and QOL after stroke, it was found that QOL is proportional to the degree of ADL performance [37]. Similarly, it has been found that patients who were independent in $\mathrm{ADL}$ had significantly better health-related quality of life than ADL-restricted patients [38]. We suggest that the degree of ADL performance could be a primary component in predicting QOL outcome.

\section{Conclusion}

In summary, PSO-SVM can perform best QOL prediction when used the combination of early outcomes of MAL_QOM and SIS as input data. MAL_QOM and SIS are likely to be predominant predictors for QOL.

The purpose of this study was to develop a computational method to identify potential predictors for quality of life (QOL) after post stroke rehabilitation. Early outcomes of MAL QOM and SIS were identified as predominant predictors for QOL. In addition, the degree of ADL performance could be a primary component in QOL prediction. The approach provides the medical team another possibility to improve the accuracy in predicting QOL in stroke patients. Therapists could determine the therapies for stroke patients more accurately and efficiently to enhance the quality of life after stroke. Larger number of patients and wider variety of treatment need to be tested with the approach to further evaluate this computational method.

\section{Acknowledgements}

The work was partly supported by Ministry of Science and Technology (NSC-97 -2314-B-002008-MY3, NSC-98-2811-B-002-015, NSC1022221-E-182-015-MY3, and MOST104-2221E-182-052-MY2), National Health Research Institutes (NHRI-EX99-9742PI and NHRIEX99-9920PI) and Chang Gung Memorial Hospital (CMRPD270053, CMRPD2C0041, CMRPD2C0042, and CMRPD2C0043) in Taiwan.

\section{References}

1. Organization WH. The Atlas of Heart Disease and Stroke, 50-51 (2004).

2. Kasner SE. Clinical interpretation and use of stroke scales, Lancet. Neurol 5(7), 603-612 (2006).

3. Duncan PW, Jorgensen HS, Wade DT. Outcome measures in acute stroke trials: A systematic review and some recommendations to improve practice. Stroke 31(6), 1429-1438 (2000).

4. Health WHO DoM, WHOQOL-BREF: Introduction, administration, scoring and generic version of the assessment: field trial version, December 1996. Geneva: World Health Organization (1996)

5. Mackenzie $A E$, Chang $A M$. Predictors of quality of life following stroke. Disabil. Rehabil 24(5), 259-265 (2002).

6. Buck D, Jacoby A, Massey A, et al. Evaluation of measures used to assess quality of life after stroke. Stroke 31(8), 2004-2010 (2000).

7. Lin KC, Chang YF, Wu CY, et al. Effects of constraint-induced therapy versus bilateral arm training on motor performance, daily functions, and quality of life in stroke survivors. Neurorehabil. Neural. Repair 23(5),
441-448 (2009).

8. Lin $\mathrm{KC}, \mathrm{Wu} C Y$, Liu JS, et al. Constraintinduced therapy versus dose-matched control intervention to improve motor ability, basic/extended daily functions, and quality of life in stroke. Neurorehabil. Neural. Repair 23(2), 160-165 (2009).

9. Van der Lee $\mathrm{JH}$, Wagenaar RC, Lankhorst GJ, et al. Forced use of the upper extremity in chronic stroke patients: results from a single-blind randomized clinical trial. Stroke 30(11), 2369-2375 (1999).

10. Wu CY, Chuang LL, Lin KC, et al. Randomized trial of distributed constraint-induced therapy versus bilateral arm training for the rehabilitation of upper-limb motor control and function after stroke. Neurorehabil. Neural. Repair 25(2), 130-139 (2011).

11. Nys GMS, van Zandvoort MJ, van der Worp $\mathrm{HB}$, et al. Early cognitive impairment predicts long-term depressive symptoms and quality of life after stroke. J. Neurol. Sci 247(2), 149156 (2006).

12. Teoh V, Sims J, Milgrom J. Psychosocial predictors of quality of life in a sample of community-dwelling stroke survivors: A longitudinal study. Top. Stroke. Rehabil 16(2), 157-166 (2009).
13. Aprile I, Piazzini DB, Bertolini C, et al. Predictive variables on disability and quality of life in stroke outpatients undergoing rehabilitation. Neurol. Sci 27(1), 40-46 (2006).

14. Jonsson AC, Lindgren I, Hallstrom B, et al. Determinants of quality of life in stroke survivors and their informal caregivers. Stroke 36(4), 803-808, 2006.

15. Mirbagheri MM, Rymer WZ. Predication of reflex recovery after stroke using quantitative assessments of motor impairment at 1 month. Conf. Proc. IEEE Eng. Med. Biol. Soc 7252-7325 (2009).

16. Fritz SL, Light KE, Clifford SN, et al. Descriptive characteristics as potential predictors of outcomes following constraint-induced movement therapy for people after stroke. Phys. Ther 86(6), 825-832 (2006).

17. Wu CY, Lin KC, Chen HC, et al. Effects of modified constraint-induced movement therapy on movement kinematics and daily function in patients with stroke: A kinematic study of motor control mechanisms. Neurorehabil. Neural. Repair 21(5), 460-466 (2007).

18. Ploughman M, Corbett D. Can forced-use therapy be clinically applied after stroke? An exploratory randomized controlled 


\section{Prediction of quality of life after stroke rehabilitation Research}

trial. Arch. Phys. Med. Rehabil 85(9), 1417-1423 (2004).

19. van 't Veer LJ, Dai H, van de Vijver MJ, et al. Gene expression profiling predicts clinical outcome of breast cancer. Nature 415(6871), 530-536 (2002)

20. Dupuy A, Simon RM. Critical review of published microarray studies for 14 cancer outcome and guidelines on statistical analysis and reporting. J. Natl. Cancer. Inst 99(2), 147157 (2007).

21. Brunnström S. Movement therapy in hemiplegia: A neurophysiological approach, Medical Dept, Harper \& Row (1970).

22. Folstein MF, Folstein SE, McHugh PR. Minimental state: A practical method for grading the cognitive state of patients for the clinician. J. Psychiatr. Res 12(3), 189-198 (1975).

23. Bohannon RW, Smith MB. Interrater reliability of a modified Ashworth scale of muscle spasticity. Phys. Ther 67(2), 206-207 (1987).

24. Duncan PW, Bode RK, Min Lai S, et al. Rasch analysis of a new stroke-specific outcome scale: The Stroke Impact Scale. Arch. Phys. Med. Rehabil 84(7), 950-963 (2003).

25. Duncan PW, Lai SM, Bode RK, et al. Stroke Impact Scale-16: A brief assessment of physical function. Neurology 60(2), 291-296 (2003).

26. Duncan PW, Wallace D, Lai SM, et al. The stroke impact scale version 2.0. Evaluation of reliability, validity, and sensitivity to change. Stroke 30(10), 2131-2140 (1999).

27. Lauridsen $\mathrm{HH}$, Hartvigsen J, Manniche $\mathrm{C}$ et al. Responsiveness and minimal clinical important difference for pain and disability instruments in low back pain patients. $B M C$. Musculoskelet. Disord 7(1), 82 (2006).

28. Jaeschke R, Singer J, Guyatt GH. Measurement of health status. Ascertaining the minimal clinically important difference. Control. Clin. Trials 10(4), 407-415 (1989).

29. Lin KC, Fu T, Wu CY, et al. Minimal detectable change and clinically important difference of the Stroke Impact Scale in stroke patients. Neurorehabil. Neural. Repair 24(5), 486-492 (2010).

30. Gladstone DJ, Danells CJ, Black SE. The fuglmeyer assessment of motor recovery after stroke: A critical review of its measurement properties. Neurorehabil. Neural. Repair 16(3), 232-240 (2002).

31. Jain A, Zongker D. Feature selection: Evaluation, application, and small sample performance. IEEE. Trans. Pattern. Analy. Mach. Intellig 19(2), 153-158 (1997).

32. Tu CJ, Li YC, Chang JY, et al. Feature Selection using PSO-SVM. IAENG Int. J. Comp. Sci 33(1) (2007).

33. Somol P, Pudil P, Novovicovc J, et al. Adaptive floating search methods in feature selection. Patt. Recog. Lett 20(11-13), 1157-1163 (1999).

34. Sun Y, Babbs CF, Delp EJ. A comparison of feature selection methods for the detection of breast cancers in mammograms: Adaptive sequential floating search vs. genetic algorithm. IEEE-EMBS $27^{\text {th }}$ Annual Int Conf Eng Med Biol Soc (2005).

35. King RB. Quality of life after stroke. Stroke 27(9), 1467-1472 (1996).

36. Wu CY, Chuang LL, Lin KC, et al. Responsiveness and validity of two outcome measures of instrumental activities of daily living in stroke survivors receiving rehabilitative therapies. Clin. Rehabil 25(2), 175-183 (2011).

37. Ahlsio B, Britton M, Murray V, et al. Disablement and quality of life after stroke. Stroke 15(5), 886-890 (1984).

38. Ramasubbu R, Patten SB. Effect of depression on stroke morbidity and mortality. Can. J. Psychiatry 48(4), 250-257 (2003). 